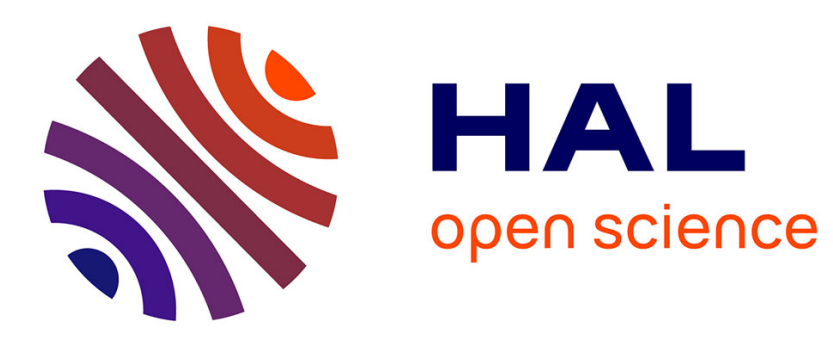

\title{
On the condensation of thick symmetric multilayer panels including dilatational motion
}

Fabien Marchetti, Uthayasuriyan Arasan, Fabien Chevillotte, Kerem Ege

\section{To cite this version:}

Fabien Marchetti, Uthayasuriyan Arasan, Fabien Chevillotte, Kerem Ege. On the condensation of thick symmetric multilayer panels including dilatational motion. Journal of Sound and Vibration, 2021, 502, pp.116078. 10.1016/j.jsv.2021.116078 . hal-03164592

\section{HAL Id: hal-03164592 https://hal.science/hal-03164592}

Submitted on 24 Mar 2021

HAL is a multi-disciplinary open access archive for the deposit and dissemination of scientific research documents, whether they are published or not. The documents may come from teaching and research institutions in France or abroad, or from public or private research centers.
L'archive ouverte pluridisciplinaire HAL, est destinée au dépôt et à la diffusion de documents scientifiques de niveau recherche, publiés ou non, émanant des établissements d'enseignement et de recherche français ou étrangers, des laboratoires publics ou privés. 


\title{
On the condensation of thick symmetric multilayer panels including dilatational motion
}

\author{
Fabien Marchetti $^{\mathrm{a}, *}$, U. Arasan $^{\mathrm{a}, \mathrm{b}, \mathrm{c}}$, Fabien Chevillotte $^{\mathrm{a}}$, \\ Kerem Ege ${ }^{\mathrm{d}}$ \\ ${ }^{a}$ Matelys - Research Lab, F-69120 Vaulx-en-Velin, France \\ ${ }^{\mathrm{b}}$ Institute for Aerospace Technology and The Composites Group, University of \\ Nottingham, University Park, NG7 2RD, UK \\ ${ }^{\mathrm{c}}$ Université de Lyon, ENTPE, LTDS UMR CNRS 5513, 3 rue Maurice Audin, 69518 \\ Vaulx-en-Velin Cedex, France \\ ${ }^{\mathrm{d}}$ Univ Lyon, INSA-Lyon, LVA, Laboratoire Vibrations Acoustique, EA677, \\ F-69621 Villeurbanne, France
}

\begin{abstract}
Condensed models are used to describe the dynamic behaviour of a multilayer structure by means of an equivalent homogeneous layer defined by intrinsic properties. Existing condensed models mainly describe the bending, membrane and shearing motions of the multilayer plate and neglect its dilatational motion. As a result, the transmission loss across the multilayer may be underestimated if the layers are soft and thick. In this paper, a condensed model of physically symmetric multilayer is developed. The antisymmetric and symmetric motions of the structure are described separately by means of two equivalent admittances. These admittances depend on three intrinsic properties: a dynamic bending stiffness and two dynamic mass densities. The condensed model is validated comparing transmission loss computations with the Transfer Matrix Method for multilayers with elastic or poroelastic cores.
\end{abstract}

Key words: condensed plate model; thick symmetric multilayer; dilatational motion; dynamic intrinsic properties; transmission loss computation

\footnotetext{
* corresponding author

Email addresses: fabien.marchetti@matelys.com (Fabien Marchetti),

arasan.uthayasuriyan@matelys.com (U. Arasan),

fabien.chevillotte@matelys.com (Fabien Chevillotte),

kerem.egedinsa-lyon. fr (Kerem Ege).
} 


\section{Introduction}

Multilayered structures have shown their interest in today's transportation and construction industries for their performances in term of high stiffness, lightweight or controlled damping that classical materials cannot offer. They are commonly used in automotive, aerospace or civil applications to lighten structures or reduce the vibration level and improve the sound insulation.

In this context, several analytical models of such structures exist in literature to describes their dynamic behaviour. Carrera [1] gives an overview and classification of most of these models. We can mention the Layer-Wise models which impose a kinematic for each layer and have a number of kinematic variables which depends on the number of layers. The model of Ghinet et al. [2] assumes a displacement field of Reissner-Mindlin [3] in each layer considering bending, membrane and shearing effects. For the special case of a three layer system, they add a dilatational term to describe the breathing (stretching) motion of the structure. Dym and Lang [4] also describe this phenomenon in their model separating the antisymmetric (bending, membrane and shearing) and symmetric (breathing) motion of the multilayer. The theory of Lamb waves [5] describes the propagation of longitudinal and transverse waves across each layer and is, therefore, part of the Layer-Wise models. The Transfer Matrix Method (TMM) [67] uses this theory to link the state variables (pressure, stresses and velocities) on both sides of each layer. The Zig-Zag models also impose one kinematic per layer but use continuity conditions at the interface between the layers to keep a fixed number of kinematic variables. In a similar way to Ghinet et al., Guyader and Lesueur [8] also assume a displacement field of Reissner-Mindlin for each layer. The model of Guyader and Lesueur, originally developed for orthotropic plates (plies aligned with the global coordinate system) and recently extended to anisotropic structures (for any orientation of plies) [9]10], can be applied on multilayer with any number of layer. Loredo [11] also developed a Zig-Zag model considering bending, membrane, shearing and dilatational effects.

All the previous models are generally applied to compute the natural wavenumbers of the structure or the transmission loss (TL) across the multilayer [12]. Other methodologies such as condensed models aim to identify an equivalent single layer which describes the behaviour of the multilayer by means of dynamic intrinsic properties. For instance, we can cite the model of Ross [13], Kerwin [14] and Ungar [15] (also known as RKU in the literature) which requires to define if the layer works in bending or in shearing. Guyader and Cacciolati [16] later adapted the previous model of Guyader and Lesueur to identify equivalent properties of isotropic multilayers. Note that this work has been recently extended to anisotropic multilayers [17]. Ege et al. [18] compared the equivalent models of RKU and Guyader with the Lamb waves model and observed consistent results in terms of equivalent stiffnesses and slightly different damping loss factors. Backström and Nilsson [19] developed a condensed model of sandwich beams with honeycomb cores and pro- 
posed different techniques to compute the apparent loss factor of the structure. Viverge et al. [20] used a homogenisation technique to analyse the different behaviours of highly contrasted laminated plates. Ruzek et al. [21] used information criteria to compare and select the optimal model from measurements. They show that simple models may be more adapted than complex models in some situations. Finally, condensed models provide multiple advantages. They can be used in finite element software to reduce the time computation substituting multilayer structures with homogeneous layers. Then, the dynamic behaviour of the multilayer is directly described through the equivalent parameter.

The condensed plate models cited previously essentially describes the bending and shearing motions of the multilayer neglecting the dilatational motion since they assume a constant normal displacement. Arasan et al. [22] have recently demonstrated that the frequency range of validity of plate models directly depends on the influence of the dilatational motion as compared to bending and shearing motions. For three layer systems with soft and thick core, such as a typical double wall partition widely used in building applications, the dilatational motion can happen at low frequencies. Therefore, we propose in this paper a condensed model of thick symmetric multilayer considering the bending, shearing and dilatational motions of the structure. The main goal of this work consists in identifying dynamic intrinsic properties to describe the behaviour of the multilayer and estimate the transmission loss using these properties. The possibility to apply the proposed condensed model on poroelastic multilayers is also addressed. The main advantage of this model is to reduce the number of degrees of freedom in FEM and thus the computational time.

The paper is divided into four parts. The first one details the TL definition using the equivalent methodology of condensed models and the TMM, which is used as a reference in this paper (TMM has already proven its relevance compared to FEM [ [23 22] $)$. The second part exhibits the limits of existing condensed models based on TL and admittances comparisons. Then, the third part presents the proposed condensed model and the equivalent intrinsic parameters. Finally, TL computations of multilayers with elastic or poroelastic cores are compared to validate the proposed condensed model.

\section{Analytical modelling of multilayer system}

\subsection{Transfer Matrix Method}

The transfer matrix method describes the wave propagation in multilayered systems. Each layer is defined by a thickness $h_{i}$ with infinite lateral dimensions and can be of different nature (fluid, elastic or poroelastic). The propagation in a layer is described by a transfer matrix $[T]$ linking the state vector $\mathcal{V}$ on both sides of the 
layer. For instance, the transfer matrix relation of layer $i$ is:

$$
\mathcal{V}\left(M_{i-1}\right)=\left[T_{i}\right] \mathcal{V}\left(M_{i}\right) .
$$

The number of waves propagating in a layer depends on its nature (one longitudinal wave for a fluid, one longitudinal and one transverse waves for a solid, two longitudinal and one transverse waves for a poroelastic). Thus, the size of the state vector and the transfer matrix depends also on the nature of the layer. The state vector of a fluid layer is:

$$
\mathcal{V}^{\mathrm{f}}=\left(\begin{array}{ll}
p & v
\end{array}\right)^{\mathrm{T}},
$$

where $p$ and $v$ are the pressure and longitudinal velocity in the fluid, respectively. The state vector of a solid layer is:

$$
\mathcal{V}^{\mathrm{s}}=\left(\begin{array}{llll}
v_{x} & v_{z} & \sigma_{z z} & \sigma_{x z}
\end{array}\right)^{\mathrm{T}},
$$

where $v_{z}$ and $v_{x}$ are longitudinal and transverse velocities and $\sigma_{z z}$ and $\sigma_{x z}$ are longitudinal and transverse stresses, respectively. Finally, the state vector of a poroelastic layer is:

$$
\mathcal{V}^{\mathrm{p}}=\left(\begin{array}{llllll}
v_{x}^{\mathrm{s}} & v_{z}^{\mathrm{s}} & v_{z}^{\mathrm{f}} & \sigma_{z z}^{\mathrm{s}} & \sigma_{x z}^{\mathrm{s}} & \sigma_{z z}^{\mathrm{f}}
\end{array}\right)^{\mathrm{T}},
$$

where the superscripts $f$ and $s$ denote the components of the waves propagating in the fluid and solid part of the poroelastic layer.

In the case of a multilayer system, the continuity between the layers is assumed to be perfect. The method describing the propagation through the whole multilayer consists in assembling the transfer matrices of each layer. Coupling matrices are used if two consecutive layers have different nature [6]. Dazel et al. [7] have shown that this method becomes unstable for high frequencies or large layer thicknesses and proposed to use the Stroh formalism to improve the stability of the method.

In this paper, we consider that the multilayer is surrounded by air and is excited by a plane acoustic wave exciting the structure at an incident angle $\theta$ (see Figure 1). The wave is defined by a transverse wavenumber:

$$
k_{\mathrm{t}}=k_{0} \sin (\theta)=\frac{\omega}{c_{0}} \sin (\theta),
$$

where $c_{0}$ corresponds to the speed of sound in the air.

Since the multilayer is surrounded by air, the state vector at the emission $\left(M_{0}\right)$ and reception $\left(M_{N}\right)$ sides of the multilayer can be defined as a state vector of a fluid. Thus, the multiplication of all the transfer matrices of the layers and the coupling 


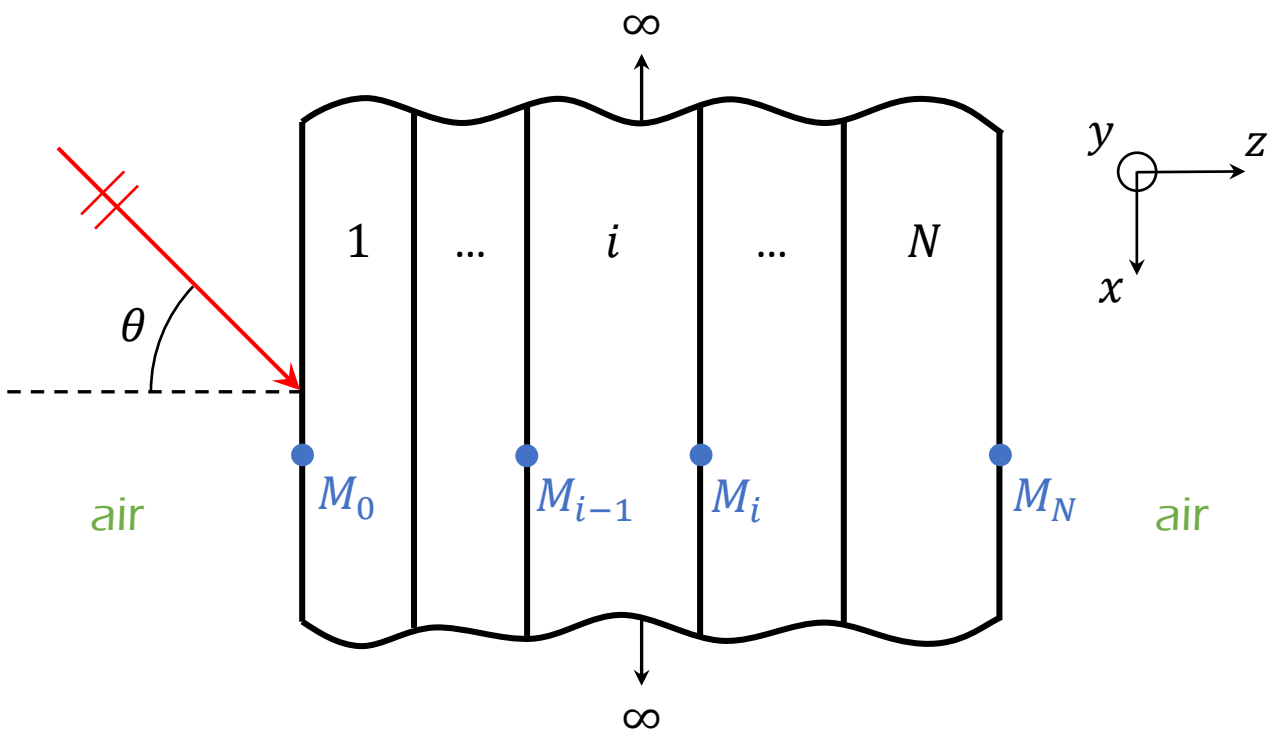

Fig. 1. Multilayer system under a plane acoustic wave excitation.

matrices leads to a $2 \times 2$ matrix relation:

$$
\left(\begin{array}{l}
p\left(M_{0}\right) \\
v\left(M_{0}\right)
\end{array}\right)=\left[\begin{array}{ll}
T_{11} & T_{12} \\
T_{21} & T_{22}
\end{array}\right]\left(\begin{array}{l}
p\left(M_{N}\right) \\
v\left(M_{N}\right)
\end{array}\right)
$$

Considering the previous matrix relation, the transmission loss (TL) across the multilayer at the incident angle $\theta$ is defined as:

$$
\mathrm{TL}(\theta)=10 \log _{10}\left(\left|\frac{1}{2}\left(T_{11}+T_{22}-\frac{T_{12}}{Z_{b}}-T_{21} Z_{b}\right)\right|^{2}\right)
$$

where $Z_{b}=\rho_{0} c_{0} / \cos (\theta)$ is the radiation impedance on the reception side and $\rho_{0}$ corresponds to the density of air.

\subsection{Existing condensed plate models}

As mentioned in the introduction, existing condensed plate models [13,14 generally describe the bending, membrane and shearing motions of each layer of the multilayer assuming that the normal displacement is constant. Then, they assume that, for a given frequency, the multilayer behaves as an equivalent plate. Thus, all the behaviour of the structure is condensed in one or more dynamic intrinsic properties which govern the equation of motion of the equivalent plate. The condensed models cited previously define the equivalent plate as a thin plate under Love-Kirchhoff's theory and the dynamic intrinsic parameter corresponds to 
a dynamic bending stiffness $\tilde{D}_{\text {eq }}(f)^{1}$. For a typical three layer system, this dynamic bending stiffness varies between a low frequency asymptote, which corresponds to the global bending of the multilayer, and a high frequency asymptote, which corresponds to the bending of the skins only [24]. The transition between these asymptotes is controlled by the shearing of the core of the multilayer. Arasan et al. [25] have recently developed a simple model to identify $\tilde{D}_{\text {eq }}(f)$ based on these asymptotes and a transition frequency. We can also cite the condensed model of Zarraga et al. [26] based on the consideration of the low-frequency bending and shear contributions. This model does not account for the high-frequency bending behavior controlled by the inner bending of the skins and does not exhibit the correct behaviour of a three-layer system at higher frequencies.

Other more complex theories could be chosen to define the behaviour of the equivalent plate such as, for instance, a Reissner-Mindlin's thick plate. As mentioned by Ruzek et al. [21], this choice may be not always adapted since it is not necessarily more accurate to use a higher number of equivalent dynamic properties.

In a similar way to the TMM, we assume that the equivalent plate is surrounded by air and excited by a plane wave defined by the transverse wavenumber $k_{\mathrm{t}}$. The transfer matrix of the equivalent plate is then defined as:

$$
\left(\begin{array}{l}
p\left(M_{0}\right) \\
v\left(M_{0}\right)
\end{array}\right)=\left[\begin{array}{cc}
1 & Z_{\mathrm{eq}} \\
0 & 1
\end{array}\right]\left(\begin{array}{l}
p\left(M_{N}\right) \\
v\left(M_{N}\right)
\end{array}\right)
$$

where $Z_{\text {eq }}$ is the impedance of the equivalent thin plate:

$$
Z_{\mathrm{eq}}=\mathrm{j} \rho h \omega\left(1-\frac{\tilde{D}_{\mathrm{eq}} k_{\mathrm{t}}^{4}}{\rho h \omega^{2}}\right)=\frac{1}{\mathrm{j} \omega}\left(\tilde{D}_{\mathrm{eq}} k_{\mathrm{t}}^{4}-\rho h \omega^{2}\right)
$$

with $h=\sum_{i} h_{i}$ the thickness and $\rho=\frac{\sum_{i} \rho_{i} h_{i}}{\sum_{i} h_{i}}$ the density of the plate. The reader may notice that $Z_{\text {eq }}$ is composed of a mass-controlled term $\left(\rho h \omega^{2}\right)$ and a stiffnesscontrolled term $\left(\tilde{D}_{\text {eq }} k_{\mathrm{t}}^{4}\right)$.

Applying Eq. (7) on the previous transfer matrix (Eq. (8)), the transmission loss of the equivalent thin plate at the incident angle $\theta$ is defined as:

$$
\mathrm{TL}(\theta)=10 \log _{10}\left(\left|1-\frac{Z_{\mathrm{eq}}}{2 Z_{b}}\right|^{2}\right) .
$$

1 The superscript $\sim$ is used in this paper to indicate that the variable is complex and depends on frequency. 


\subsection{Diffuse field computation}

For a diffuse field excitation between the angles $\theta_{\min }$ and $\theta_{\max }$, the transmission loss across the structure is derived from the following integral:

$$
\mathrm{TL}_{\mathrm{df}}=-10 \log _{10}\left(\frac{\int_{\theta_{\min }}^{\theta_{\max }} 10^{-\mathrm{TL}(\theta) / 10} \cos (\theta) \sin (\theta) \mathrm{d} \theta}{\int_{\theta_{\min }}^{\theta_{\max }} \cos (\theta) \sin (\theta) \mathrm{d} \theta}\right)
$$

The diffuse field computations presented in this paper are calculated between the angles 0 and $\pi / 2$.

\section{Limitations of existing condensed plate models}

This section aims to identify the limits of the existing condensed models taking as reference the TMM. In the following paragraphs, the transmission loss computed from the TMM and the equivalent model of Guyader and Cacciolati [16] are compared for two different multilayer system. Then, the antisymmetric and symmetric admittances of the multilayer are analysed to explain the differences between the models.

\subsection{Transmission loss}

Figure 2 compares the transmission loss of a sandwich plate computed from the TMM and the equivalent plate model. The studied sandwich plate is composed of steel skins and a core sufficiently rigid to avoid dilatational effects but still soft enough to exhibit shearing effects. The material properties of the layers are written in Table 1. The transmission is globally controlled by the mass law at low frequency and strongly decreases at the coincidence frequency, which corresponds to the frequency at which the transverse wavenumber $k_{\mathrm{t}}$ is equal to the bending wavenumber. We can observe that, for this configuration of sandwich, the equivalent model gives similar results as the TMM up to high frequency $(f<10 \mathrm{kHz})$ and correctly estimates the coincidence frequency of the multilayer $(f \approx 5.2 \mathrm{kHz})$.

A similar comparison is presented in Figure 3 for a second sandwich composed of the same steel skins and a very soft core. The elastic properties of the layers are summarized in Table 1. We can observe that, for this case, the TL computed from the TMM has, in addition to the coincidence frequency, another singularity 
Table 1

\begin{tabular}{l|c|c|c} 
& Skins $($ steel $)$ & Stiff core & Soft core \\
\hline Thickness $h(\mathrm{~mm})$ & 3 & 3 & 3 \\
Density $\rho\left(\mathrm{kg} \cdot \mathrm{m}^{-3}\right)$ & 7800 & 1000 & 55 \\
Young's modulus $E(\mathrm{GPa})$ & 210 & 0.2 & 0.000043 \\
Poisson's ratio $\nu(-)$ & 0.33 & 0.48 & 0.4 \\
Damping $\eta(-)$ & 0.03 & 0.05 & 0.3
\end{tabular}

Elastic properties of the isotropic layers used in this paper.

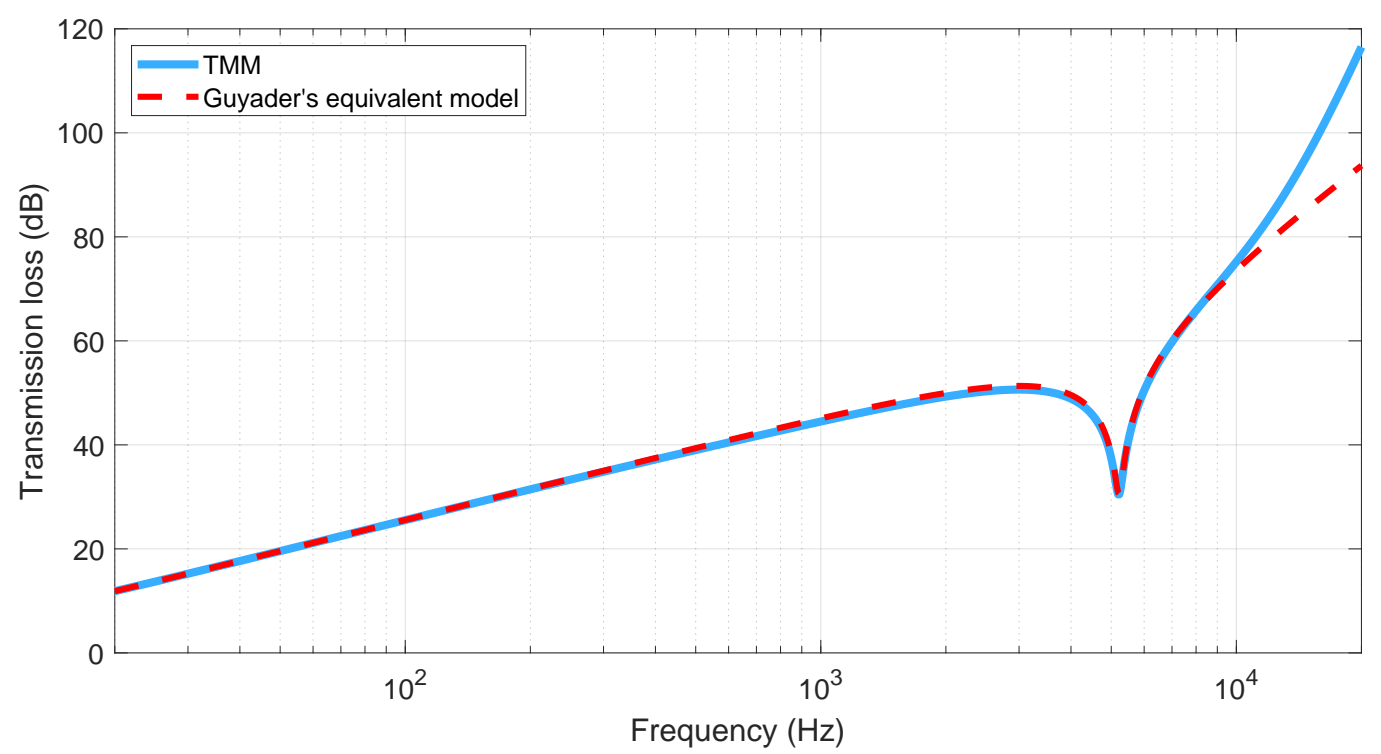

Fig. 2. Transmission loss of the steel/stiff core/steel sandwich computed using the TMM (solid blue line) and Guyader's equivalent model (dashed red line). See Table 1 for the material properties of the layers. Analytical simulations calculated at $60^{\circ}$ of incidence.

at a lower frequency $(f \approx 260 \mathrm{~Hz}$ ). This singularity corresponds to the massspring-mass resonance of the multilayer and is called the breathing frequency. This physical phenomenon is not described by the condensed model since the equivalent methodology assumes a constant normal velocity. As a consequence, the TL estimated by the equivalent plate model is strongly underestimated after the breathing frequency. We can still notice that the coincidence frequency is correctly estimated by the equivalent model.

\subsection{Symmetric and antisymmetric admittances}

As stated in the section 2.2, existing condensed plate models essentially describe the flexural, membrane and shearing waves propagating in the structure by means of dynamic intrinsic parameters. This type of waves are defined by an antisymmetric 


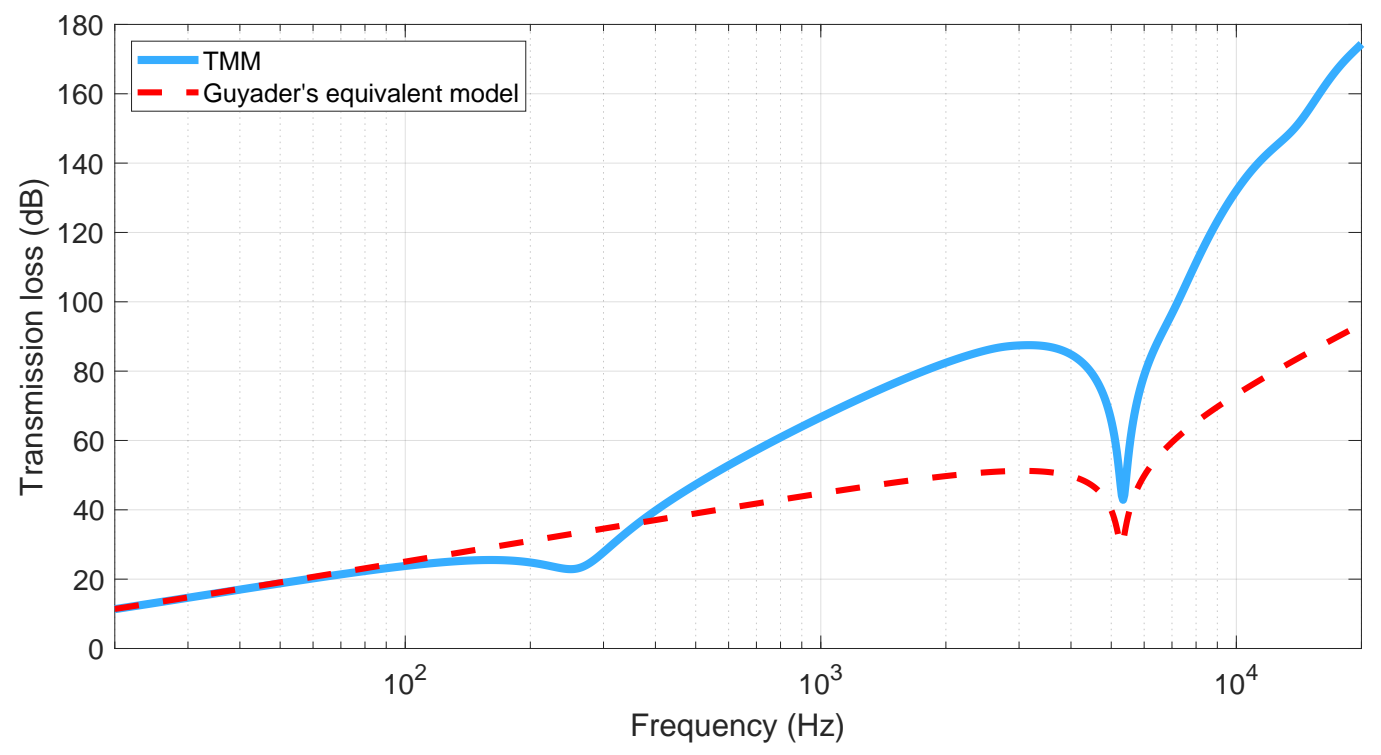

Fig. 3. Transmission loss of the steel/soft core/steel sandwich computed using the TMM (solid blue line) and Guyader's equivalent model (dashed red line). See Table 1 for the material properties of the layers. Analytical simulations calculated at $60^{\circ}$ of incidence.

motion of the plate (see Figure 4a). On the contrary, existing condensed plate models neglect the breathing or stretching motion of the system, which is considered as a symmetric motion (see Figure 4b), since they assume no deformation in the thickness. In order to understand the differences between the condensed model and the full modelling of the multilayers with the TMM, this section is focused on the analysis of the antisymmetric and symmetric motions of the structure comparing the admittances of both motions.

(a) Antisymmetric wave

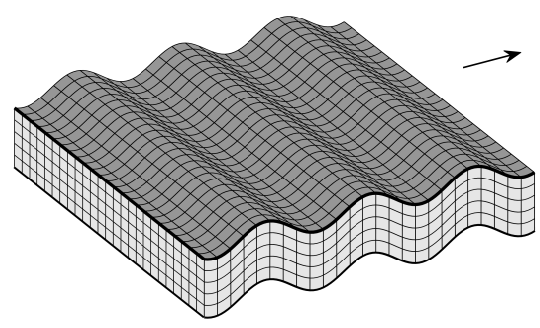

(b) Symmetric wave

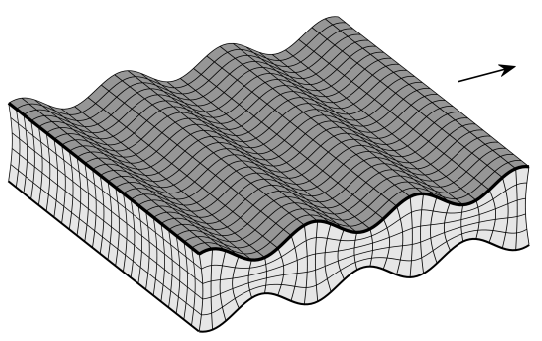

Fig. 4. Antisymmetric (a) and symmetric (b) waves propagating in a plate.

According to Dym and Lang [427], the symmetric and antisymmetric motions are uncoupled for physically symmetric multilayer panels. This assumption is valid since the coupling components of each symmetric layers are balanced and thus can be neglected in the computation of the kinematic and deformation energies of 
the system. Thus, both behaviours can be separately described by means of the symmetric $\left(Z_{\mathrm{S}}\right)$ and antisymmetric $\left(Z_{\mathrm{A}}\right)$ impedances of the system. Dym and Lang define these impedances as functions of the pressure and velocity at the emission and reception sides of the multilayer:

$$
\begin{gathered}
Z_{\mathrm{S}}=-\frac{p\left(M_{0}\right)+p\left(M_{N}\right)}{\bar{v}}=2 \frac{p\left(M_{0}\right)+p\left(M_{N}\right)}{v\left(M_{0}\right)-v\left(M_{N}\right)}, \\
Z_{\mathrm{A}}=\frac{p\left(M_{0}\right)-p\left(M_{N}\right)}{\tilde{v}}=2 \frac{p\left(M_{0}\right)-p\left(M_{N}\right)}{v\left(M_{0}\right)+v\left(M_{N}\right)},
\end{gathered}
$$

where $\bar{v}$ and $\tilde{v}$ correspond to the symmetric and antisymmetric normal velocities. Since these definitions are only valid for physically symmetric multilayer, only this type of multilayer is studied in this paper. From these definitions, the transfer matrix of the multilayer can be defined as function of the antisymmetric $\left(Y_{\mathrm{A}}=1 / Z_{\mathrm{A}}\right)$ and symmetric $\left(Y_{\mathrm{S}}=1 / Z_{\mathrm{S}}\right)$ admittances:

$$
\left(\begin{array}{l}
p\left(M_{0}\right) \\
v\left(M_{0}\right)
\end{array}\right)=\left[\begin{array}{cc}
\frac{Y_{\mathrm{A}}+Y_{\mathrm{S}}}{Y_{\mathrm{A}}-Y_{\mathrm{S}}} & \frac{1}{Y_{\mathrm{A}}-Y_{\mathrm{S}}} \\
\frac{4 Y_{\mathrm{A}} Y_{\mathrm{S}}}{Y_{\mathrm{A}}-Y_{\mathrm{S}}} & \frac{Y_{\mathrm{A}}+Y_{\mathrm{S}}}{Y_{\mathrm{A}}-Y_{\mathrm{S}}}
\end{array}\right]\left(\begin{array}{l}
p\left(M_{N}\right) \\
v\left(M_{N}\right)
\end{array}\right) .
$$

Comparing Eq. (6) and Eq. (14), the symmetric and antisymmetric admittances can be expressed as functions of the terms of the transfer matrix of the TMM:

$$
\begin{aligned}
& Y_{\mathrm{S}}=\frac{T_{11}-1}{2 T_{12}}, \\
& Y_{\mathrm{A}}=\frac{T_{11}+1}{2 T_{12}} .
\end{aligned}
$$

As an example, Figure 5 presents the antisymmetric and symmetric admittances of the steel/stiff core/steel sandwich calculated with the TMM at $60^{\circ}$ of incidence using Eq. (15) and Eq. (16). We can observe that the antisymmetric admittance globally decreases with frequency except at the coincidence frequency of the structure. On the contrary, the symmetric admittance increases with frequency until reaching the antisymmetric admittance around $10 \mathrm{kHz}$ depending on the angle of incidence. Before $10 \mathrm{kHz}, Y_{\mathrm{S}}$ is negligible as compared to $Y_{\mathrm{A}}$ meaning that the behaviour of the structure is mostly governed by the antisymmetric motion in this frequency band. After $10 \mathrm{kHz}$, both admittances are of the same order of magnitude and the symmetric motion can not be neglected. The results of the TMM are also compared to the equivalent admittance $\left(Y_{\mathrm{eq}}=1 / Z_{\mathrm{eq}}\right)$ of Guyader's model (Eq. (9). We can see that $Y_{\text {eq }}$ follows particularly well the antisymmetric admittance computed from the TMM on the whole frequency band. The differences observed on the TL in Figure 2 after $10 \mathrm{kHz}$ can be explained by the fact that the equivalent model does not take into account $Y_{\mathrm{S}}$, which becomes non-negligible in this frequency range. Such 
analysis of admittances has already been used to estimate the frequency range of validity of plates model [22].

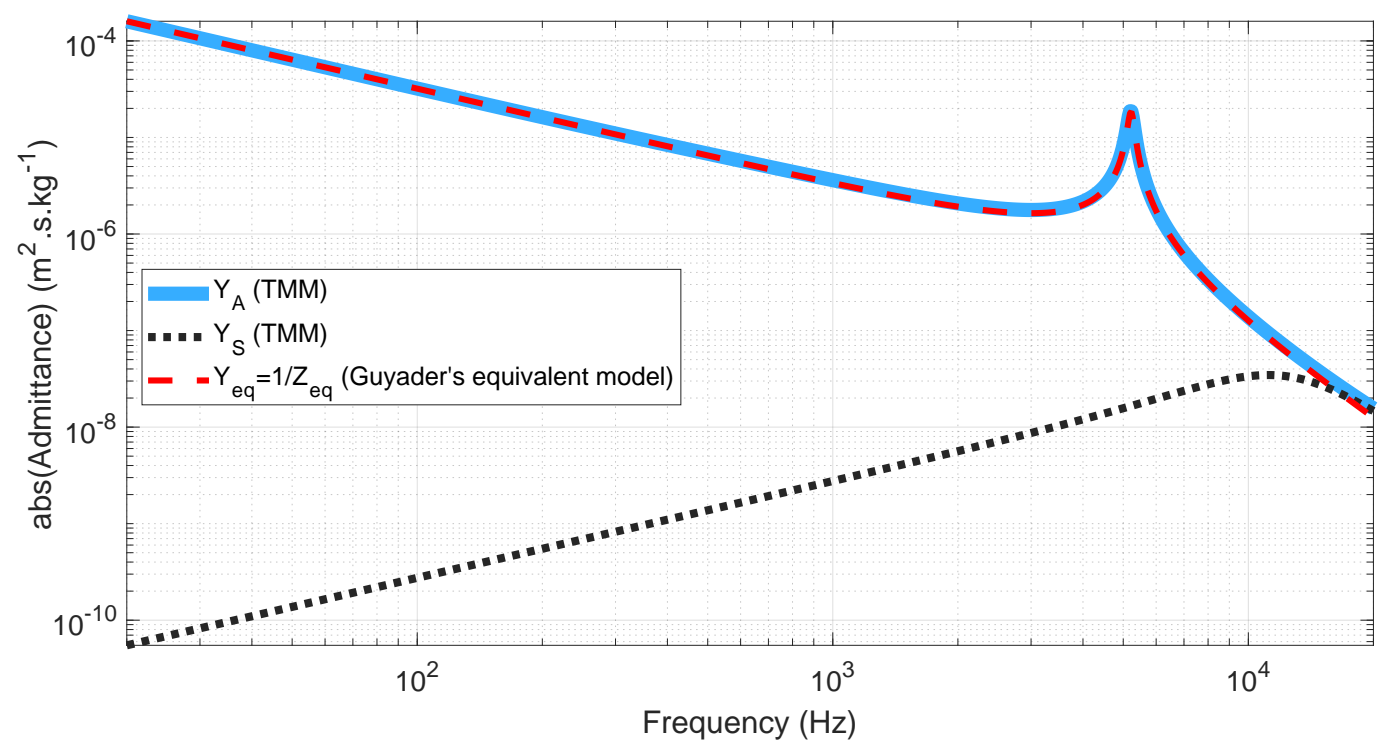

Fig. 5. Absolute values of the antisymmetric (solid blue line) and symmetric (dotted black line) admittances of the steel/stiff core/steel sandwich computed with the TMM. The dashed red line corresponds to the equivalent admittance computed using Guyader's model. The elastic properties of the layers are summarized in Table 1. Analytical simulations calculated at $60^{\circ}$ of incidence.

A similar comparison is presented in Figure 6 for the second sandwich. In this case, the symmetric admittance increases up to the breathing frequency of the system and then decreases until following the antisymmetric admittance. As a consequence, the coincidence frequency is also visible on the symmetric admittance. To explain this phenomenon, we can study the Lamb waves model [5] which uses the same theory of elasticity than the TMM. The only difference between the two approaches is that the TMM imposes an acoustic wave defined by the transverse wavenumber $k_{\mathrm{t}}$ and the Lamb waves model identifies the natural wavenumbers of the multilayer in vacuum. Figure 7 presents the dispersion curves of the zeroth-order antisymmetric $\left(\mathrm{A}_{0}\right)$ and symmetric $\left(\mathrm{S}_{0}\right)$ motions of the second sandwich identified from the Lamb waves model. The transverse wavenumber imposed by the TMM is also drawn for an incidence of $60^{\circ}$. We can observed that the $S_{0}$ curve has a cut-on frequency and tends to the $\mathrm{A}_{0}$ curve at higher frequency. The singularities observed on the TL and the admittances appear at frequencies where $k_{\mathrm{t}}$ crosses the natural wavenumbers of the multilayer (i.e. breathing and coincidence frequencies for the symmetric motion and coincidence frequency for the antisymmetric motion). Since $A_{0}$ and $S_{0}$ curves are close to each other at high frequency, the coincidence frequencies of both motions are similar.

We can also observe on Figure 6 that the equivalent admittance is again very close to the antisymmetric admittance computed from the TMM meaning that the con- 
densed plate model gives an accurate description of the antisymmetric motion even for sandwich structures with a soft core. Finally, the symmetric motion can not be neglected after $150 \mathrm{~Hz}$, which corresponds to the frequency where the TL computed from the equivalent model of Guyader and Cacciolati begins to deviate from the solution of the TMM (see Figure 3).

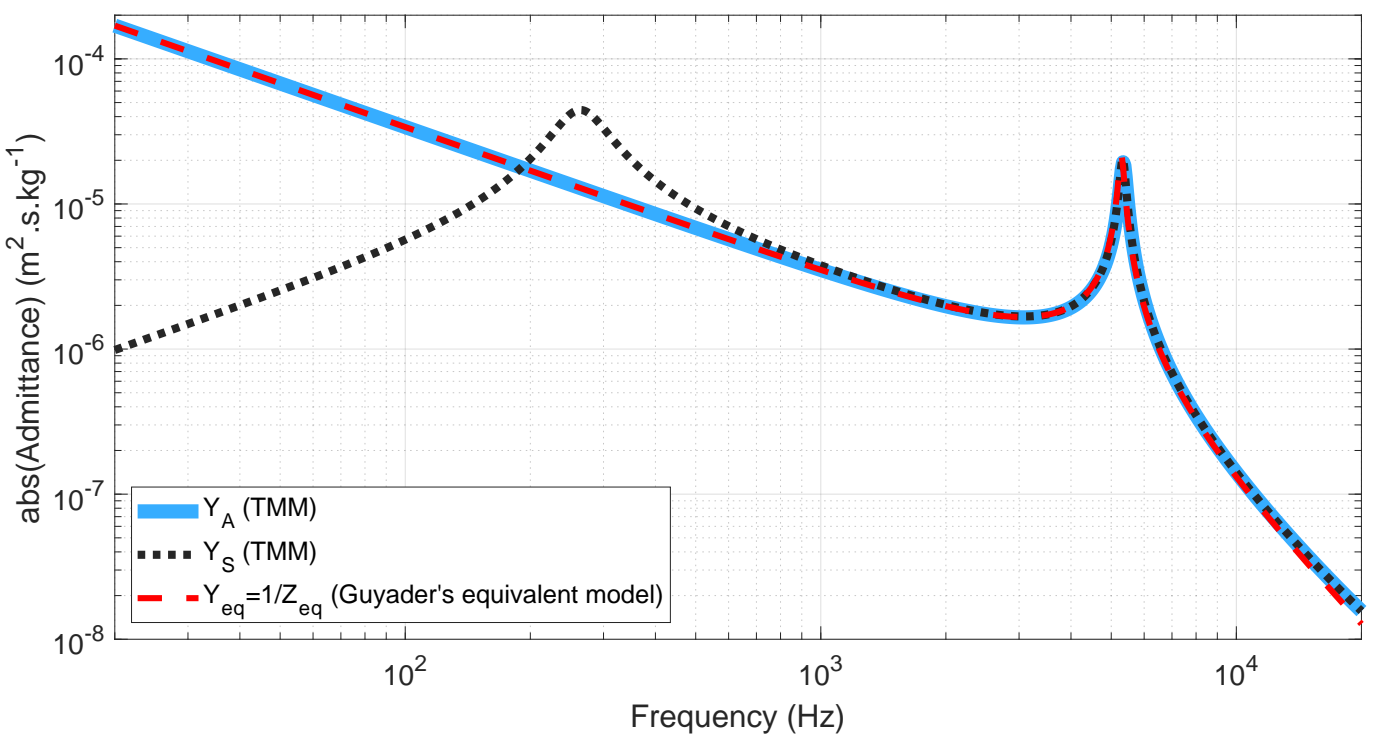

Fig. 6. Absolute values of the antisymmetric (solid blue line) and symmetric (dotted black line) admittances of the steel/soft core/steel sandwich computed with the TMM. The dashed red line corresponds to the equivalent admittance computed using Guyader's model. The elastic properties of the layers are summarized in Table 1 . Analytical simulations calculated at $60^{\circ}$ of incidence.

In the case of an excitation at normal incidence $(\theta=0)$, the coincidence frequency tends toward infinity for both admittances (see Figure. 8). The antisymmetric motion is then essentially controlled by the mass law of the system while the symmetric motion is controlled by the dilatational effect of the layers with the massspring-mass resonance. The reader may also notice that the breathing frequency is identical to the $60^{\circ}$ case since it does not depend on the angle of incidence. Thus, the low frequency behaviours of the symmetric and antisymmetric admittances are similar at every angle of incidence.

Based on the previous observations, we can conclude that condensed plate models correctly describe the antisymmetric motion of the multilayer but are incomplete when the symmetric motion becomes significant since they assume no deformation in the thickness. 


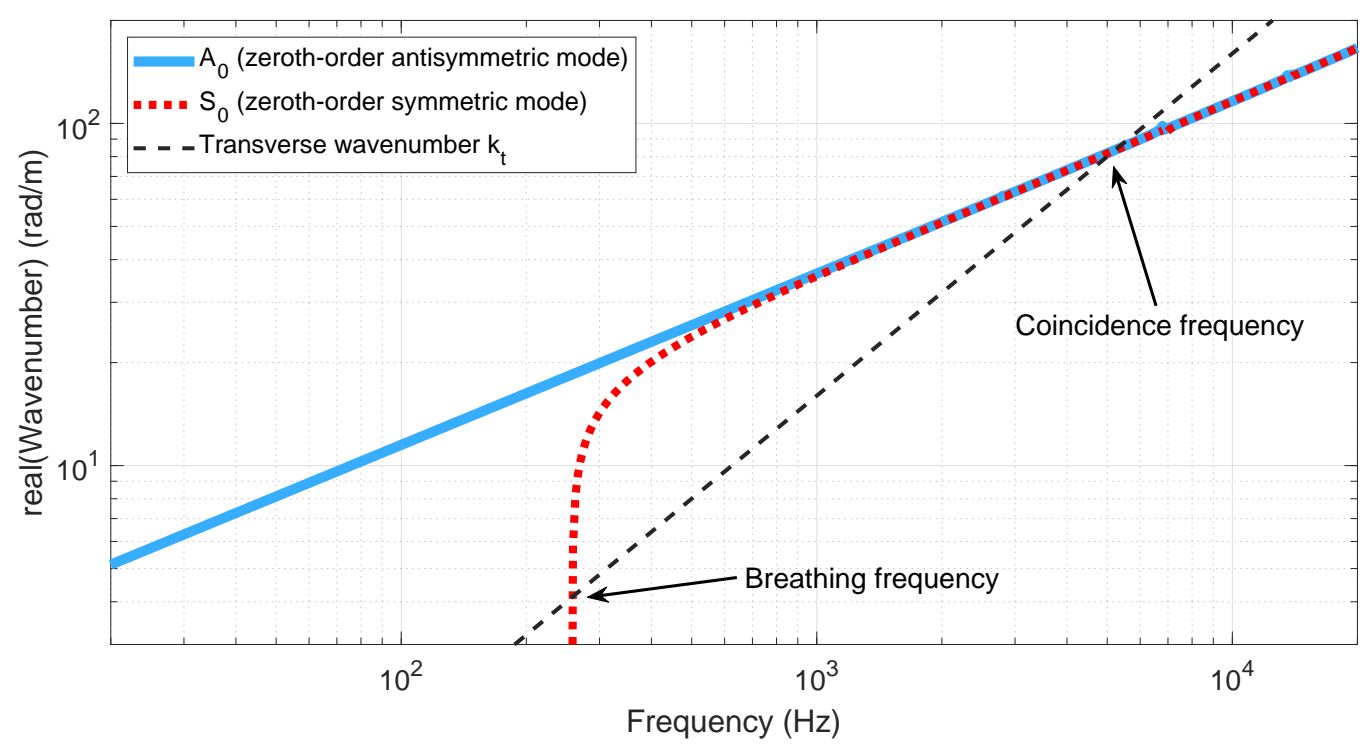

Fig. 7. Real part of the dispersion curves of the zeroth-order antisymmetric (solid blue line) and symmetric (dotted red line) motions of the steel/soft core/steel sandwich (see the elastic properties of the layers in Table 1] obtained from the Lamb waves model [5]. The dashed black line corresponds to the transverse wavenumber of the plane wave at $60^{\circ}$ of incidence.

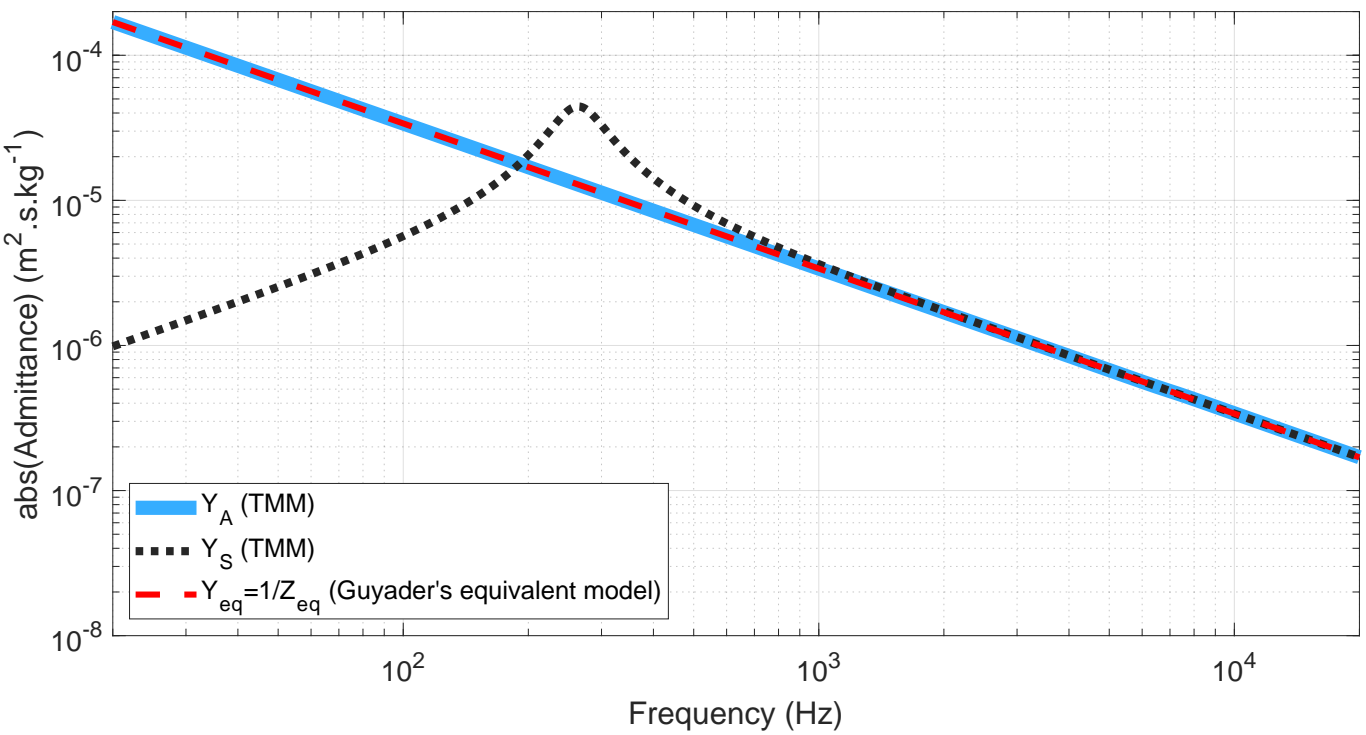

Fig. 8. Absolute values of the antisymmetric (solid blue line) and symmetric (dotted black line) admittances of the steel/soft core/steel sandwich computed with the TMM. The dashed red line corresponds to the equivalent admittance computed using Guyader's model. The elastic properties of the layers are summarized in Table 1 . Analytical simulations calculated at normal incidence. 


\section{Proposed condensed model}

This section focuses on the development of a condensed model of physically symmetric multilayer panels considering both the antisymmetric and symmetric motions of the structure. In order to describe these motions, equivalent admittances are defined by means of intrinsic properties.

\subsection{Equivalent admittances}

We observed in section 3.2 that the antisymmetric and symmetric admittances have a similar coincidence frequency, which is well estimated by the condensed plate model. In this model, the coincidence frequency essentially depends on the stiffness-controlled term $\tilde{D}_{\text {eq }} k_{\mathrm{t}}^{4}$. Based on these observations, we could also use $\tilde{D}_{\text {eq }} k_{\mathrm{t}}^{4}$ to identify the coincidence frequency of the symmetric motion. We also noticed that the differences between the symmetric and antisymmetric admittances essentially occur in the low frequency range around the breathing frequency. Considering that the breathing frequency does not depend on the angle of incidence and thus on $k_{\mathrm{t}}$ as we observed in section 3.2, this phenomenon can be described by an intrinsic property that also does not depend on the transverse wavenumber $k_{\mathrm{t}}$. In the plate model, such parameter corresponds to the mass density of the impedance. Based on these observations, we could assume that the breathing frequency of the symmetric motion is controlled by a dynamic mass density $\tilde{\rho}_{\mathrm{s}}$. Finally, the symmetric impedance of the multilayer can be derived from the impedance of an equivalent thin plate of Love-Kirchhoff defined by a dynamic mass and a dynamic stiffness:

$$
Z_{\mathrm{S}, \mathrm{eq}}=\frac{1}{\mathrm{j} \omega}\left(\tilde{D}_{\mathrm{eq}} k_{\mathrm{t}}^{4}-\tilde{\rho}_{\mathrm{S}} h \omega^{2}\right) .
$$

We also observed that the equivalent admittance computed from the condensed plate model gives similar results to the antisymmetric admittance of the multilayer computed from the TMM. By analogy with the equivalent symmetric impedance (17), we can increase the accuracy of the condensed model by considering an antisymmetric dynamic mass density $\tilde{\rho}_{\mathrm{A}}$ in the equivalent impedance:

$$
Z_{\mathrm{A}, \mathrm{eq}}=\frac{1}{\mathrm{j} \omega}\left(\tilde{D}_{\mathrm{eq}} k_{\mathrm{t}}^{4}-\tilde{\rho}_{\mathrm{A}} h \omega^{2}\right) .
$$

The advantages of $\tilde{\rho}_{\mathrm{A}}$ as compared to the theoretical mass density $\rho$ are discussed and illustrated in sections 4.3 and 5 .

From the definition of Eq. (18) and Eq. (17), the following equivalent admittances 
can be derived:

$$
Y_{\mathrm{A}, \text { eq }}=\frac{1}{Z_{\mathrm{A}, \mathrm{eq}}} \quad ; \quad Y_{\mathrm{S}, \mathrm{eq}}=\frac{1}{Z_{\mathrm{S}, \mathrm{eq}}}
$$

Finally, the coefficients $\tilde{\rho}_{\mathrm{A}}, \tilde{\rho}_{\mathrm{S}}$ and $\tilde{D}_{\text {eq }}$ correspond to the dynamic intrinsic properties of the proposed condensed model since they do not depend on the angle of incidence of the imposed acoustic wave. Doing so, we assume that the breathing frequency of the multilayer, which is described by $\tilde{\rho}_{\mathrm{S}}$, does not depend on the angle of incidence as we observed in section 3.2 .

\subsection{Identification of the equivalent intrinsic parameters}

The equivalent flexural rigidity $\tilde{D}_{\text {eq }}$ can be identified from the existing condensed plate model that we already discussed in section 2.2. In this paper, we use the model of Guyader and Cacciolati [16] to identify this parameter. Note that RKU model [13 14 15] or the simple three-layer model recently proposed by Arasan et al. [25] could be used as well. Concerning the identification of the dynamic mass densities $\tilde{\rho}_{\mathrm{S}}$ and $\tilde{\rho}_{\mathrm{A}}$, two strategies are proposed in the following subsections.

\subsubsection{Transfer matrix identification at normal incidence}

The first strategy consists in calculating the transfer matrix at normal incidence. Then, the equivalent admittances (Eq. (19) ) can be identified from this matrix using Eq. (15) and Eq. (16). At normal incidence, the equivalent admittances only depend on the mass densities since $k_{\mathrm{t}}=0$. Thus, the dynamic mass densities are defined as:

$$
\tilde{\rho}_{\mathrm{A}}=\frac{1}{\mathrm{j} h \omega Y_{\mathrm{A}}(\theta=0)} \quad ; \quad \tilde{\rho}_{\mathrm{S}}=\frac{1}{\mathrm{j} h \omega Y_{\mathrm{S}}(\theta=0)}
$$

This method has the advantages that it can be applied on physically symmetric multilayer composed of any number and type (fluid, solid, poroelastic) of layers. Dilatational motions inside the layers are considered since the solid and poroelastic layers are not modeled as plates in the TMM.

\subsubsection{Mass-spring-mass simplified identification}

The second strategy has been developed for sandwich structures, which are composed of two stiff skins and a soft and light core. The symmetric motion of this type of multilayer excited at normal incidence can be described by a simple massspring-mass system [28]. The masses correspond to the skins (which are supposed to be identical since the multilayer is physically symmetric) and are defined by a surface mass $\mathcal{M}=\rho_{1} h_{1}=\rho_{3} h_{3}$. The spring corresponds to the core and is defined 
by a compliance $\mathcal{C}$. If the core is solid, the compliance is $\mathcal{C}_{\text {solid }}=h_{2} /\left(\lambda_{2}+2 \mu_{2}\right)$ where $\lambda_{2}=E_{2} \nu_{2} /\left(\left(1+\nu_{2}\right)\left(1-2 \nu_{2}\right)\right)$ and $\mu_{2}=E_{2} /\left(2\left(1+\nu_{2}\right)\right)$ are the Lamé coefficients of the core. If the core is poroelastic, the compliance can either be calculated using the model of Biot [29]30] or can be estimated by adding the stiffnesses of the fluid and the solid in parallel. Thus, the compliance is $\mathcal{C}_{\text {porous }}=$ $\mathcal{C}_{\text {fluid }} \mathcal{C}_{\text {solid }} /\left(\mathcal{C}_{\text {fluid }}+\mathcal{C}_{\text {solid }}\right)$, where $\mathcal{C}_{\text {fluid }}$ is the compliance of the fluid and can be approximated by $\mathcal{C}_{\text {fluid }}=h_{2} / P_{0}$ at low frequency for high porosity (close to 1 ), with $P_{0}$ the atmospheric pressure.

Using this simple mass-spring-mass system, the transfer matrix at normal incidence can be written as:

$$
\begin{aligned}
& \left(\begin{array}{l}
p\left(M_{0}\right) \\
v\left(M_{0}\right)
\end{array}\right)=\left[\begin{array}{cc}
1 & \mathrm{j} \omega \mathcal{M} \\
0 & 1
\end{array}\right]\left[\begin{array}{cc}
1 & 0 \\
\mathrm{j} \omega \mathcal{C} & 1
\end{array}\right]\left[\begin{array}{cc}
1 & \mathrm{j} \omega \mathcal{M} \\
0 & 1
\end{array}\right]\left(\begin{array}{l}
p\left(M_{N}\right) \\
v\left(M_{N}\right)
\end{array}\right), \\
& \left(\begin{array}{l}
p\left(M_{0}\right) \\
v\left(M_{0}\right)
\end{array}\right)=\left[\begin{array}{cc}
1-\omega^{2} \mathcal{C} \mathcal{M} & \mathrm{j} \omega\left(2 \mathcal{M}-\omega^{2} \mathcal{C} \mathcal{M}^{2}\right) \\
\mathrm{j} \omega \mathcal{C} & 1-\omega^{2} \mathcal{C} \mathcal{M}
\end{array}\right]\left(\begin{array}{l}
p\left(M_{N}\right) \\
v\left(M_{N}\right)
\end{array}\right) .
\end{aligned}
$$

Using the definition of the symmetric admittance (Eq. (15)) on the previous matrix relation (Eq. (22) ) and the equivalent definition of the symmetric impedance (Eq. (17)) at normal incidence $\left(k_{\mathrm{t}}=0\right)$, the dynamic mass density $\tilde{\rho}_{\mathrm{S}}$ can be defined as:

$$
\tilde{\rho}_{\mathrm{S}}=-\frac{4 \mathcal{M}-2 \omega^{2} \mathcal{C} \mathcal{M}^{2}}{h \omega^{2} \mathcal{C} \mathcal{M}}=2 \frac{\mathcal{M}}{h}\left(1-\frac{2}{\omega^{2} \mathcal{C M}}\right) .
$$

Concerning the antisymmetric motion, the theoretical mass density is used as the dynamic mass density: $\tilde{\rho}_{\mathrm{A}}=\rho$. In this case, the equivalent antisymmetric impedance corresponds to the impedance of usual condensed plate models $\left(Z_{\mathrm{A}, \mathrm{eq}}=Z_{\mathrm{eq}}\right)$.

Finally, the mass-spring-mass assumption gives simple expressions of the dynamic mass densities. Note that this method can be applied on a multilayer system with any number of layers considering the mass $\mathcal{M}_{i}$ and the compliance $\mathcal{C}_{i}$ of each layer $i$. Doing so, the transfer matrix can be written as:

$$
\left(\begin{array}{l}
p\left(M_{0}\right) \\
v\left(M_{0}\right)
\end{array}\right)=\left[\begin{array}{cc}
1 & \mathrm{j} \omega \mathcal{M}_{1} \\
\mathrm{j} \omega \mathcal{C}_{1} & 1
\end{array}\right] \ldots\left[\begin{array}{cc}
1 & \mathrm{j} \omega \mathcal{M}_{i} \\
\mathbf{j} \omega \mathcal{C}_{i} & 1
\end{array}\right] \ldots\left[\begin{array}{cc}
1 & \mathrm{j} \omega \mathcal{M}_{N} \\
\mathrm{j} \omega \mathcal{C}_{N} & 1
\end{array}\right]\left(\begin{array}{l}
p\left(M_{N}\right) \\
v\left(M_{N}\right)
\end{array}\right),
$$




\subsection{Comparison of the dynamic mass densities}

Figure 9 compares the absolute values of the dynamic mass densities computed on the steel/soft material/steel sandwich using the two strategies described in section 4.2. The antisymmetric mass density $\tilde{\rho}_{\mathrm{A}}$ is relatively constant on the whole frequency band and closed to the theoretical mass density $\rho$. The symmetric mass density $\tilde{\rho}_{\mathrm{S}}$ decreases at low frequency until the breathing frequency and then increases until reaching the theoretical mass density $\rho$. Both strategies give similar results on the whole frequency band but some differences can be noticed at high frequencies: the higher-order resonances (located in the core) are not considered by the mass-spring-mass assumption since this method only described the first resonance at the breathing frequency.

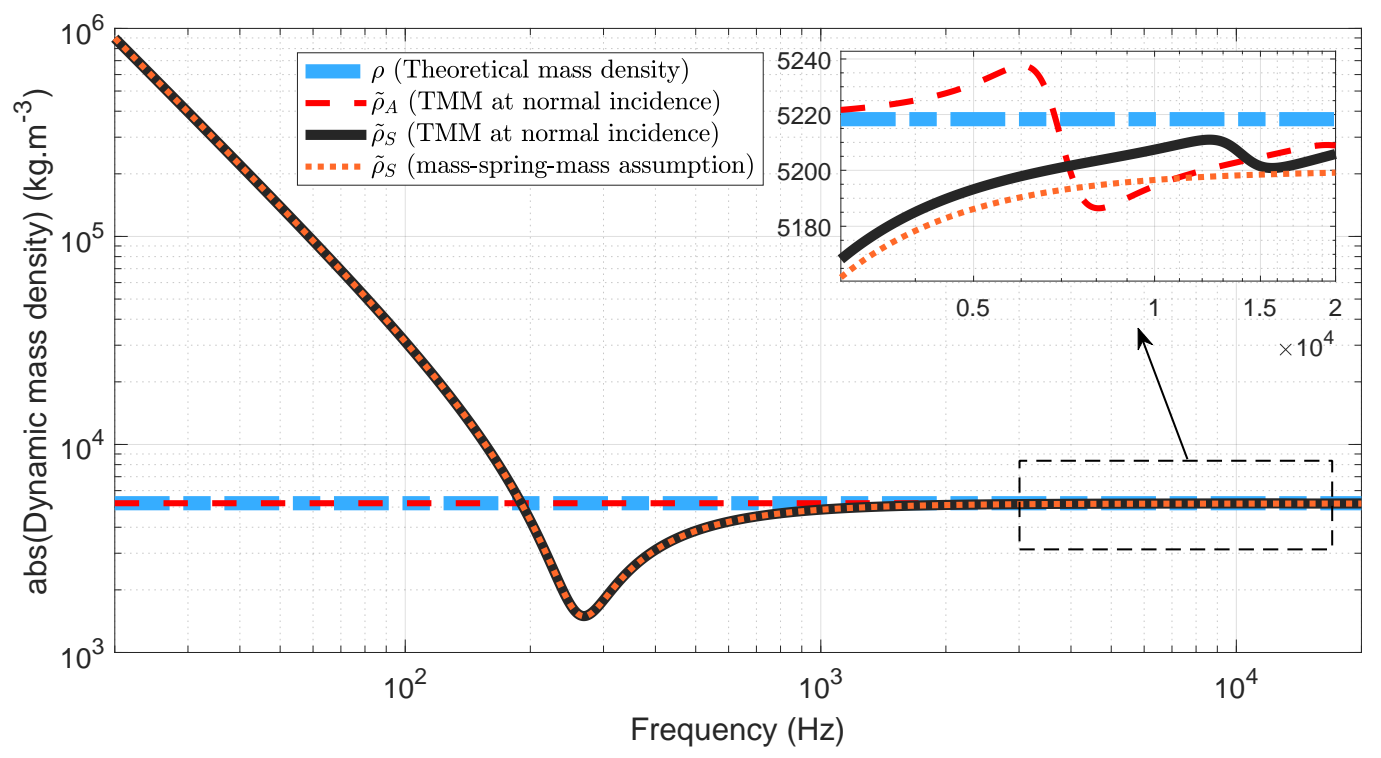

Fig. 9. Absolute values of the dynamic mass densities $\tilde{\rho}_{\mathrm{A}}$ and $\tilde{\rho}_{\mathrm{S}}$ of the steel/soft core/steel sandwich computed using the mass-spring-mass assumption (orange dotted line) and the TMM at normal incidence (red dashed and solid black lines). The blue dashed-dotted line corresponds to the theoretical mass density. See Table 1 for the material properties of the layers.

\section{Validation}

The proposed condensed model can estimate the transfer matrix (Eq. (14)) and the TL (Eq. (7)) of a given multilayered plate at each angle of incidence by means of the antisymmetric and symmetric equivalent admittances (Eq (19)). These admittances are derived from three dynamic intrinsic properties $\left(\tilde{D}_{\text {eq }}, \tilde{\rho}_{\mathrm{A}}\right.$ and $\left.\tilde{\rho}_{\mathrm{S}}\right)$ that do not depend on the angle of incidence. The flexural rigidity $\tilde{D}_{\text {eq }}$ can be identified from existing condensed models. The dynamic mass densities $\tilde{\rho}_{\mathrm{A}}$ and $\tilde{\rho}_{\mathrm{S}}$ can be 
identified using one computation of the TMM approach at normal incidence (see section 4.2.1) or the mass-spring-mass assumption (see section 4.2.2).

In order to validate that the intrinsic parameters are able to take into account the effect of incident angle, this section is dedicated to the comparison between the proposed condensed model and the TMM using TL computations under diffuse field.

\subsection{Three layer system with soft elastic core}

Figure 10 compares the TL of the steel/soft material/steel sandwich computed in diffuse field with the TMM and the proposed equivalent model using the two strategies to identify the dynamic mass densities. We can observe that the breathing frequency of the multilayer is correctly described by the equivalent approach. The results are very close to the estimations of the TMM on the whole frequency band using the TMM at normal incidence to calculate the dynamic mass densities. The mass-spring-mass assumption gives consistent results up to $5 \mathrm{kHz}$ where the higher resonances appear. Figure 10 also presents the TL calculated using the contribution of the antisymmetric (assuming $Y_{\mathrm{S}}=0$ ) and symmetric (assuming $Y_{\mathrm{A}}=0$ ) motions separately. We can observed that none of contributions can be used alone meaning that both contributions should be considered simultaneously to correctly estimate the TL.

\subsection{Three layer system with poroelastic core}

A similar comparison to the one of section 5.1 is presented in Figure 11 for a steel/polyurethane/steel sandwich. The polyurethane layer is a poroelastic material which has been described with the Biot's model [29:30] and the JohnsonChampoux-Allard [31|32] model for the visco-thermal dissipation in the TMM. This latter model depends on five acoustic properties (open porosity, airflow resistivity, viscous characteristic length, thermal characteristic length and high frequency limit of tortuosity). The values of these parameters are summarized in Table 2. The same elastic properties as the soft material are used. Concerning the mass-spring-mass assumption, the polyurethane layer has been considered either as a solid and a poroelastic material using the approximations detailed in section 4.2.2. We can observe that the mass-spring-mass assumption gives incorrect results if only a solid compliance is used for the polyurethane layer. The acoustic parameters of the polyurethane are not considered using this assumption. As a consequence, the breathing frequency is not correctly estimated. On the contrary, good results are obtained with the mass-spring-mass assumption considering a poroelastic compliance. Using the TMM at normal incidence to calculate the mass densities, the condensed model gives a correct estimation of the TL. The separate contributions 


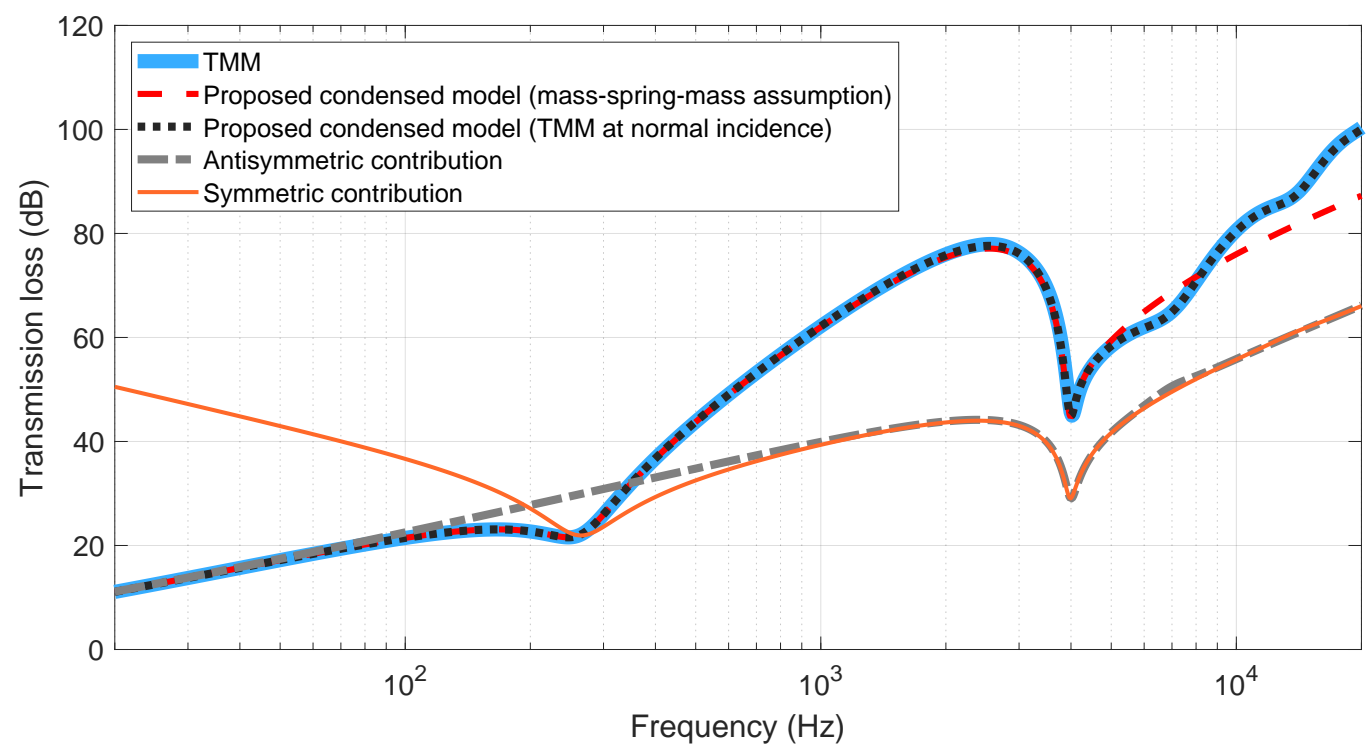

Fig. 10. Transmission loss of the steel/soft material/steel sandwich computed using the TMM (thick solid blue line), the proposed condensed model with the mass-spring-mass assumption (dashed red line) and the TMM at normal incidence (dotted black line). The dotted-dashed gray and thin solid orange lines correspond to the contribution of the antisymmetric and symmetric motions respectively. See Table 1 for the material properties of the layers. Analytical simulations calculated in diffuse field.

of the symmetric and antisymmetric motions are also drawn in Figure 11 and lead to the same conclusions as the previous paragraph. Two additional remarks can be noticed. First, the equivalent flexural rigidity $\tilde{D}_{\text {eq }}$ is still calculated using elastic parameters only. Secondly, the breathing frequency and its resonances may change with the angle of incidence for low resistivity values $\left(\sigma<1000\right.$ N.s.m $\left.{ }^{-4}\right)$. Thus, the proposed model would not be valid for these low values of resistivity.

Table 2

\begin{tabular}{l|c} 
Open porosity $\phi(-)$ & 0.97 \\
\hline Airflow resistivity $\sigma\left(\right.$ N.s.m $\left.^{-4}\right)$ & 50000 \\
\hline Viscous characteristic length $\Lambda(\mu \mathrm{m})$ & 60 \\
\hline Thermal characteristic length $\Lambda^{\prime}(\mu \mathrm{m})$ & 160 \\
\hline High frequency limit of the tortuosity $\alpha_{\infty}(-)$ & 1.1
\end{tabular}

Acoustic properties of the polyurethane layer.

\section{Conclusion}

In this paper, a condensed model of physically symmetric multilayer is developed considering the antisymmetric and symmetric motion of the system. Both motions 


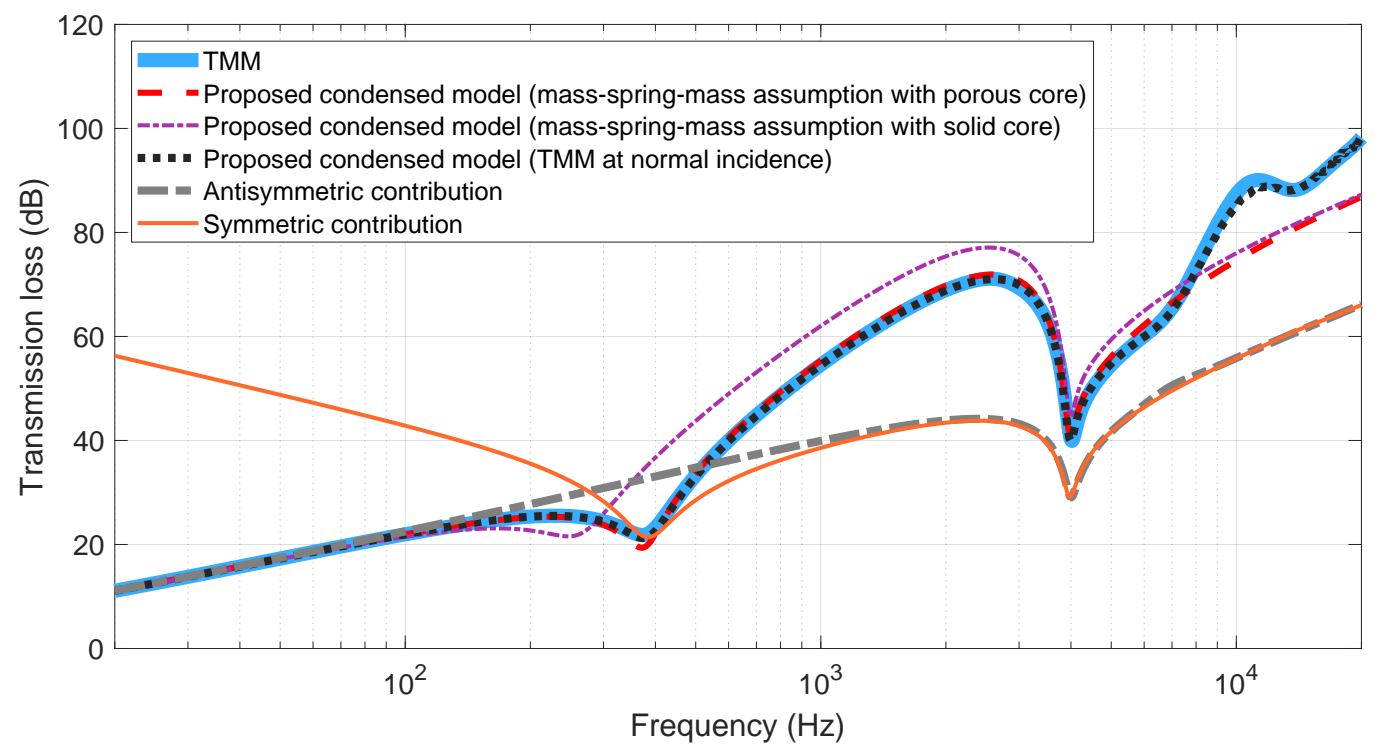

Fig. 11. Transmission loss of the steel/polyurethane/steel sandwich computed using the TMM (thick solid blue line), the proposed condensed model with the mass-spring-mass assumption (dashed red line and dotted-dashed purple thin line) and the TMM at normal incidence (dotted black line). The dotted-dashed gray and thin solid orange lines correspond to the contribution of the antisymmetric and symmetric motions respectively. See Table 1 for the elastic properties of the layers and Table 2 for the acoustic properties of the polyurethane. Analytical simulations calculated in diffuse field.

are described separately by means of the admittances of two equivalent thin plates. It is worth mentioning that the antisymmetric (mainly bending, shearing) and the symmetric (compression) motions can be described by three intrinsic properties: a dynamic bending stiffness, which corresponds to the equivalent parameter identified from existing condensed plate model, and two dynamic mass densities. Two different strategies are developed to identify the dynamic mass densities. The first one uses the TMM approach at normal incidence to describe the breathing frequency of the system and its resonances. This method, which can be applied with any type (fluid, solid and poroelastic) and number of layers, shows very good results as compared to the full multilayer modelling with the TMM in diffuse field. The second strategy describes the multilayer excited at normal incidence as a massspring-mass system. This method gives a simple description of the breathing frequency but neglects the higher resonances. Using the proposed condensed model, the transfer matrix linking the pressure and velocity on both sides of the multilayer can be estimated at different angles of incidence by means of the intrinsic properties. Finally, the proposed condensed model gives an accurate estimation of the transmission loss describing at the same time the antisymmetric and symmetric motions of the structure.

The first application of this work could be to use the condensed model in finite element framework. A dedicated shell element can be developed to describe the antisymmetric and symmetric motions of the multilayer by means of the identified 
intrinsic properties. This condensed model should considerably reduce the computational time compared to a full 3D modelling of the multilayer. This FEM implementation is underwork. Another perspective could be to extend this condensed model to asymmetric multilayers.

\section{Acknowledgments}

The authors would like to gratefully acknowledge Marie-Skłodowska Curie Actions (MSCA) Project 765472 'N2N: No2Noise' for financial support. This work was performed within the framework of the Labex CeLyA of Universite de Lyon, operated by the French National Research Agency (ANR-10-LABX-0060/ ANR11-IDEX-0007).

\section{References}

[1] E. Carrera, An assessment of mixed and classical theories on global and local response of multilayered orthotropic plates, Composite Structures 50 (2) (2000) 183-198.

[2] S. Ghinet, N. Atalla, Modeling thick composite laminate and sandwich structures with linear viscoelastic damping, Computers and Structures 89 (15) (2011) 1547 - 1561. doi:https://doi.org/10.1016/j.compstruc.2010.09.008.

[3] R. Mindlin, Influence of rotary inertia and shear on flexural motions of isotropic, elastic plates, Journal of Applied Mechanics 18 (1951) 31-38.

[4] C. L. Dym, M. A. Lang, Transmission of sound through sandwich panels, The Journal of the Acoustical Society of America 56 (5) (1974) 1523-1532.

[5] H. Lamb, On waves in an elastic plate, Proceedings of the Royal Society of London A: Mathematical, Physical and Engineering Sciences 93 (648) (1917) 114 - 128.

[6] B. Brouard, D. Lafarge, J.-F. Allard, A general method of modelling sound propagation in layered media, Journal of Sound and Vibration 183 (1) (1995) 129 - 142. doi:https://doi.org/10.1006/jsvi.1995.0243

[7] O. Dazel, J.-P. Groby, B. Brouard, C. Potel, A stable method to model the acoustic response of multilayered structures, Journal of Applied Physics 113 (8) (2013) 083506. doi:10.1063/1.4790629.

[8] J. L. Guyader, C. Lesueur, Acoustic transmission through orthotropic multilayered plates, part 1: Plate vibration modes, Journal of Sound and Vibration 58 (1978) 51-68.

[9] R. L. Woodcock, Free vibration of advanced anisotropic multilayered composites with arbitrary boundary conditions, Journal of Sound and Vibration 312 (4) (2008) 769 788. doi:https://doi.org/10.1016/j.jsv.2007.11.015 
[10] A. Loredo, A. Castel, A multilayer anisotropic plate model with warping functions for the study of vibrations reformulated from Woodcock's work, Journal of Sound and Vibration 332 (1) (2013) 102 - 125. doi: https://doi.org/10.1016/j. jsv.2012.07.033.

[11] A. Loredo, A multilayered plate theory with transverse shear and normal warping functions, Composite Structures 156 (2016) 361 - 374. doi : https : / / doi . org/ $10.1016 / j$.compstruct.2015.08.084.

[12] J. Guyader, C. Lesueur, Acoustic transmission through orthotropic multilayered plates, part ii: Transmission loss, Journal of Sound and Vibration 58 (1) (1978) 69 - 86. doi:https://doi.org/10.1016/s0022-460X(78)80061-3.

[13] D. Ross, Damping of plate flexural vibrations by means of viscoelastic laminae, Structural Damping (1959) 49-97.

[14] E. M. Kerwin Jr, Damping of flexural waves by a constrained viscoelastic layer, The Journal of the Acoustical Society of America 31 (7) (1959) 952-962.

[15] E. E. Ungar, E. M. Kerwin Jr, Loss factors of viscoelastic systems in terms of energy concepts, The Journal of the Acoustical Society of America 34 (7) (1962) 954-957.

[16] J. L. Guyader, C. Cacciolati, Viscoelastic properties of single layer plate material equivalent to multi-layer composites plate, Internoise (2007) 1558-1567.

[17] F. Marchetti, K. Ege, Q. Leclère, N. Roozen, On the structural dynamics of laminated composite plates and sandwich structures; a new perspective on damping identification, Journal of Sound and Vibration 474 (2020) 115256. doi:https: //doi.org/10.1016/j.jsv.2020.115256.

[18] K. Ege, N. Roozen, Q. Leclère, R. G. Rinaldi, Assessment of the apparent bending stiffness and damping of multilayer plates; modelling and experiment, Journal of Sound and Vibration 426 (2018) 129 - 149. doi : https://doi.org/10.1016/ j.jsv.2018.04.013.

[19] D. Backström, A. Nilsson, Modelling the vibration of sandwich beams using frequency-dependent parameters, Journal of Sound and Vibration 300 (3) (2007) 589 -611. doi:https://doi.org/10.1016/j.jsv.2006.07.048

[20] K. Viverge, C. Boutin, F. Sallet, Model of highly contrasted plates versus experiments on laminated glass, International Journal of Solids and Structures 102-103 (2016) 238 -258. doi:https://doi.org/10.1016/j.ijsolstr.2016.09.035.

[21] M. Ruzek, J.-L. Guyader, C. Pézerat, Information criteria and selection of vibration models, The Journal of the Acoustical Society of America 136 (6) (2014) 3040-3050. doi:10.1121/1.4900562.

[22] U. Arasan, F. Marchetti, F. Chevillotte, G. Tanner, D. Chronopoulos, E. Gourdon, On the accuracy limits of plate theories for vibro-acoustic predictions, Journal of Sound and Vibration (2020) 115848doi:https://doi.org/10.1016/j. jsv.2020.115848. 
[23] D. Rhazi, N. Atalla, Transfer matrix modeling of the vibroacoustic response of multimaterials structures under mechanical excitation, Journal of Sound and Vibration 329 (13) (2010) 2532-2546. doi:https://doi.org/10.1016/j.jsv. 2010.01 .013 .

[24] F. Fahy, P. Gardonio, Sound and Structural Vibration, 2nd Edition, Academic Press, Oxford, 2007. doi:https://doi.org/10.1016/B978-012373633-8/ $50007-7$

[25] U. Arasan, F. Marchetti, F. Chevillotte, L. Jaouen, D. Chronopoulos, E. Gourdon, A simple equivalent plate model for dynamic bending stiffness of three-layer sandwich panels with shearing core, Journal of Sound and Vibration (2021) 116025doi: https://doi.org/10.1016/j.jsv.2021.116025.

[26] O. Zarraga, I. Sarría, J. García-Barruetabeña, F. Cortés, Homogenised formulation for plates with thick constrained viscoelastic core, Computers \& Structures 229 (2020) 106185. doi:10.1016/j.compstruc.2019.106185.

[27] C. L. Dym, C. S. Ventres, M. A. Lang, Transmission of sound through sandwich panels: A reconsideration, The Journal of the Acoustical Society of America 59 (2) (1976) 364-367.

[28] J. Davy, Sound transmission of cavity walls due to structure borne transmission via point and line connections, The Journal of the Acoustical Society of America 132 (2012) 814-21.

[29] M. A. Biot, Theory of propagation of elastic waves in a fluid-saturated porous solid. i. low-frequency range, J. Acoust. Soc. Am. 28 (2) (1956) 168-178.

[30] M. A. Biot, Theory of propagation of elastic waves in a fluid-saturated porous solid. ii. higher frequency range, J. Acoust. Soc. Am. 28 (2) (1956) 179-191.

[31] D. L. Johnson, J. Koplik, R. Dashen, Theory of dynamic permeability and tortuosity in fluid-saturated porous media, J. Fluid Mech. 176 (1987) 379-402.

[32] Y. Champoux, J.-F. Allard, Dynamic tortuosity and bulk modulus in air-saturated porous media, J. Appl. Phys. 70 (1991) 1975-1979. 


\section{List of Figures}

$1 \quad$ Multilayer system under a plane acoustic wave excitation.

2 Transmission loss of the steel/stiff core/steel sandwich computed using the TMM (solid blue line) and Guyader's equivalent model (dashed red line). See Table 1 for the material properties of the layers. Analytical simulations calculated at $60^{\circ}$ of incidence.

3 Transmission loss of the steel/soft core/steel sandwich computed using the TMM (solid blue line) and Guyader's equivalent model (dashed red line). See Table 1 for the material properties of the layers. Analytical simulations calculated at $60^{\circ}$ of incidence.

$4 \quad$ Antisymmetric (a) and symmetric (b) waves propagating in a plate.

5 Absolute values of the antisymmetric (solid blue line) and

\begin{tabular}{|l|}
\hline symmetric (dotted black line) admittances of the steel/stiff \\
\hline \hline core/steel sandwich computed with the TMM. The dashed \\
\hline \hline red line corresponds to the equivalent admittance computed \\
\hline \hline using Guyader's model. The elastic properties of the layers are \\
\hline \hline summarized in Table 1 1. Analytical simulations calculated at $60^{\circ}$ of \\
\hline incidence.
\end{tabular}

6 Absolute values of the antisymmetric (solid blue line) and symmetric (dotted black line) admittances of the steel/soft core/steel sandwich computed with the TMM. The dashed red line corresponds to the equivalent admittance computed using Guyader's model. The elastic properties of the layers are summarized in Table 1 . Analytical simulations calculated at $60^{\circ}$ of incidence.

$7 \quad$ Real part of the dispersion curves of the zeroth-order antisymmetric (solid blue line) and symmetric (dotted red line) motions of the steel/soft core/steel sandwich (see the elastic properties of the layers in Table 1) obtained from the Lamb waves model [5]. The dashed black line corresponds to the transverse wavenumber of the plane wave at $60^{\circ}$ of incidence. 
8 Absolute values of the antisymmetric (solid blue line) and

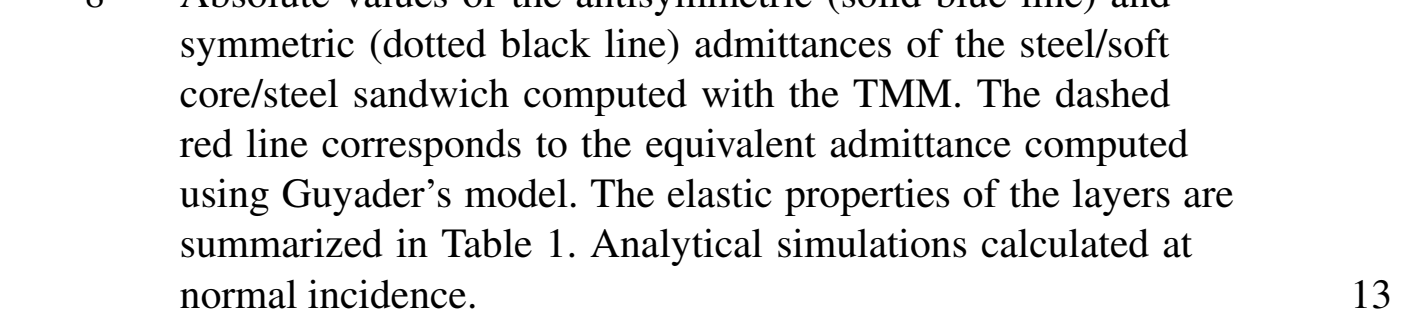

$9 \quad$ Absolute values of the dynamic mass densities $\tilde{\rho}_{\mathrm{A}}$ and $\tilde{\rho}_{\mathrm{S}}$ of the steel/soft core/steel sandwich computed using the mass-spring-mass assumption (orange dotted line) and the TMM at normal incidence (red dashed and solid black lines). The blue dashed-dotted line corresponds to the theoretical mass density. See Table|1|for the material properties of the layers. 17

10 Transmission loss of the steel/soft material/steel sandwich computed using the TMM (thick solid blue line), the proposed condensed model with the mass-spring-mass assumption (dashed red line) and the TMM at normal incidence (dotted black line). The dotted-dashed gray and thin solid orange lines correspond to the contribution of the antisymmetric and symmetric motions respectively. See Table 1 for the material properties of the layers. Analytical simulations calculated in diffuse field.

11 Transmission loss of the steel/polyurethane/steel sandwich computed using the TMM (thick solid blue line), the proposed condensed model with the mass-spring-mass assumption (dashed red line and dotted-dashed purple thin line) and the TMM at normal incidence (dotted black line). The dotted-dashed gray and thin solid orange lines correspond to the contribution of the antisymmetric and symmetric motions respectively. See Table 1 for the elastic properties of the layers and Table 2 /for the acoustic properties of the polyurethane. Analytical simulations calculated in diffuse field. 


\section{List of Tables}

$1 \quad$ Elastic properties of the isotropic layers used in this paper. 8

\begin{tabular}{lll}
\hline 2 & Acoustic properties of the polyurethane layer. & 19
\end{tabular} 